\title{
Efeito de aditivos no comportamento reológico do rejeito da bauxita
}

\section{(Effect of additives in the rheologic behavior of the bauxite tailing)}

\author{
S. G. Gonçalves, K. C. Ferreira, S. M. Brum, J. A. S. Souza, A. M. P. F. Felipe \\ Laboratório de Reologia, Instituto de Tecnologia, Universidade Federal do Pará, R. Augusto Corrêa s/n, \\ Belém, PA 66075-110 \\ silvane.goncalves@itec.ufpa.br,keyllafcastro@yahoo.com.br,sebastiao.brum@vale.com, \\ jass@ufpa.br,ampf@ufpa.br
}

\begin{abstract}
Resumo
Na mineração da bauxita no município de Paragominas-PA, são descartados no processo de beneficiamento rejeitos com teor de sólido de 33,5\% e granulometria muito fina com $\mathrm{D}_{50}$ abaixo de $5 \mu \mathrm{m}$. Esses fatores contribuem para que esta suspensão formada basicamente por silico-aluminatos, com elevada concentração de argila caulinitica, apresente aumento na viscosidade e na tensão inicial de escoamento, fatores importantes durante o processo de bombeamento. Foi realizada análise granulométrica e determinadas as composições químicas e mineralógicas do material. Avaliou-se a utilização dos aditivos hexametafosfato de sódio e poliacrilamida $25 \%$ aniônica visando à diminuição da viscosidade. Os resultados mostram diminuição da viscosidade com a adição de $23,8 \mathrm{~g} /$ ton para a poliacrilamida e 101,75 g/ton para o hexametafosfato de sódio. A granulometria da polpa de bauxita descartada e o $\mathrm{pH}$ influenciam no comportamento dos aditivos reológicos.
\end{abstract}

Palavras-chave: aditivos, rejeitos de bauxita, reologia.

Abstract

In the bauxite mining in Parogominas, PA, Brazil, tailings are discarded in the processing with 33.5\% of solids content and small particle size with $D_{50}$ below $5 \mu \mathrm{m}$. These factors contribute to this suspension basically formed by silicoaluminates, with high concentrations of kaolinite clay, increase the viscosity and the yield stress, these factors are important during the pumping. It was performed granulometric analysis and determined the chemical and mineralogical composition of the material. It was studied the use of the sodium hexametaphosphate and polyacrylamide $25 \%$ anionic to decrease the viscosity. The result show that the viscosity decrease with use of $23.8 \mathrm{~g} /$ ton for the polyacrylamide and $101.75 \mathrm{~g} /$ ton for the sodium hexametaphosphate. The particle size and the $p H$ have influence in the behavior of the additives.

Keywords: additives, bauxite tailings, rheology.

\section{INTRODUÇÃO}

Nas atividades de mineração de bauxita no município de Paragominas - Brasil, o beneficiamento é feito por meio da britagem e lavagem da bauxita bruta, a fim de remover argila e areia fina. A retirada dessa fração, que é considerada resíduo de lavagem, é descartada como rejeito com baixa porcentagem de sólidos em barragens. Atualmente a produção de bauxita e de 9,9 milhões de toneladas anuais e o descarte de resíduo da lavagem na mina representa cerca de $25 \%$ do material lavrado $[1,2]$.

A superfície de partículas sólidas minerais caracterizase pela existência de cargas positivas e negativas. Quanto mais finas as partículas, maior será sua área superficial com consequente aumento nas cargas elétricas. Dependendo do $\mathrm{pH}$, o meio apresentará uma densidade superficial de cargas positivas ou negativas. Quanto maior a densidade de cargas superficiais, maior será a espessura da dupla camada elétrica, assim como a energia de repulsão resultante entre as partículas ocasionando a dispersão da suspensão. A esse mecanismo dá-se o nome de repulsão eletrostática [3-5].
A utilização de moléculas poliméricas para auxiliar na estabilização de suspensões caracteriza a estabilização estérica. E o mecanismo combinado da estabilização eletrostática e estérica é denominado estabilização eletroestérica.

O grau de dissociação com consequente adsorção dos polieletrólitos é dependente das interações eletrostáticas entre eles e a superfície das partículas. Sendo assim, a química da superfície de uma fase sólida e as propriedades da solução dos polieletrólitos são parâmetros importantes controlados pelo $\mathrm{pH}$ e pela força iônica, sendo que a cadeia assume conformação estendida quando o grau de dissociação é elevado, e adquirem conformação enovelada quando o grau de dissociação é baixo [6, 7].

A capacidade dos aditivos em modificar as propriedades reológicas representa uma oportunidade para melhorar o funcionamento e desempenho dos processos minerais, bem como melhorar o transporte, reduzindo os gastos com energia e evitando possíveis paradas durante o bombeamento.

Neste trabalho foi estudada a viabilidade de utilização do hexametafosfato de sódio e poliacrilamida aniônica (Figs. 1a e 1b) como dispersantes do rejeito da lavagem da bauxita, 


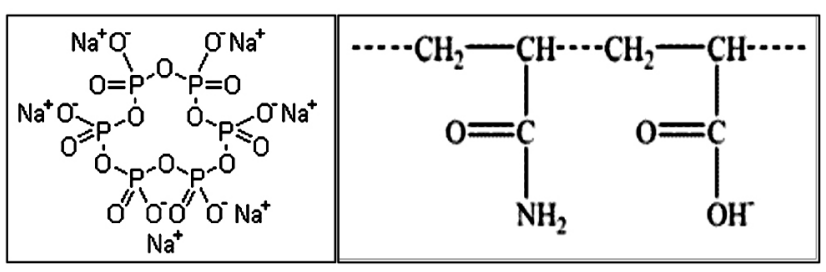

Figura 1: a) Estrutura cíclica do NaHMP (hexametafosfato de sódio) [8]; b) Estrutura molecular da poliacrilamida aniônica [9]. [Figure 1: a) Ciclic structure of the NaHMP (sodium hexametaphosphate) [8]; b) Molecular structure of the anionic polyacrylamide [9].]

contribuindo para a melhora no processo de bombeamento na mina de bauxita Paragominas no estado do Pará, Brasil.

\section{MATERIAIS E MÉTODOS}

A amostra de rejeito de bauxita é oriunda da usina de beneficiamento da mina de bauxita de Paragominas PA, com teor de sólido de 33,5\% em massa e pH 6,5.

A análise granulométrica foi realizada por peneiramento a úmido em um conjunto suspenso de malhas da escala Tyler e para as frações finas utilizou-se técnica da difração a laser em dois modelos Mastersizer2000, Malvern Instr..

A determinação da composição química foi feita por espectrometria de fluorescência de raios X, em espectrômetro WDS sequencial, Axios Minerals. As aquisições e tratamento dos dados foram feitos no software Super QManager. Para a determinação do conteúdo de $\mathrm{Al}_{2} \mathrm{O}_{3}$ aproveitável e de $\mathrm{SiO}_{2}$ reativa utilizou-se a metodologia adotada em [10].

A análise de difração de raios $X(D R X)$ foi feita no difratômetro X'Pert Pro MPD (PW 3040/60). O resultado foi interpretado no programa X'PertHighScore.

Utilizou-se o viscosímetro Haake VT 550 com sensor tipo cilindros coaxiais SV1 para ensaios de viscosidade. A programação foi taxa de cisalhamento de $100 \mathrm{~s}^{-1} \mathrm{em} 20 \mathrm{~s}$.

Amostras de rejeito com teor de sólido de $33,5 \%$ em base seca foram submetidas às análises de viscosidades em diferentes condições. Para avaliar o efeito do $\mathrm{pH}$ na formação de cargas e no comportamento dos aditivos, determinou-se a viscosidade em amostras em diferentes $\mathrm{pH}$. $\mathrm{O}$ aditivo hexametafosfato de sódio (ARCsul) foi utilizado em amostras com pH 6,5 oriundas da mina. Utilizou-se ainda a poliacrilamida $25 \%$ aniônica (Ashland), floculante na faixa de pH 6-10). A poliacrilamida $25 \%$ aniônica foi usada em rejeito com $\mathrm{pH}$ 6,5 e em amostras previamente ajustadas para $\mathrm{pH} 8,5$, com o objetivo de avaliar a influência do $\mathrm{pH}$ no desempenho do aditivo. As variações de $\mathrm{pH}$ durante as sucessivas adições foram mínimas (menor que 0,1\%); portanto, não foram consideradas neste trabalho. Foi avaliado a influência da granulometria na ação do aditivo; para tanto foram realizados ensaios em amostras passante na malha de 400\# Tyler, correspondendo a granulometria inferior a $38 \mu \mathrm{m}$.

\section{RESULTADOS E DISCUSSÃO}

As Tabelas I e II apresentam a composição química
Tabela I - Caracterização química em óxidos do rejeito. [Table I - Chemical characterization of the oxides of the tailing.]

\begin{tabular}{cc}
\hline Composição & \% em massa \\
\hline $\mathrm{Al}_{2} \mathrm{O}_{3 \text { total }}$ & 42,54 \\
$\mathrm{Al}_{2} \mathrm{O}_{3 \text { aproveitável }}$ & 21,28 \\
$\mathrm{SiO}_{2 \text { total }}$ & 22,00 \\
$\mathrm{SiO}_{2 \text { reativa }}$ & 20,03 \\
$\mathrm{TiO}_{2}$ & 1,75 \\
$\mathrm{Fe}_{2} \mathrm{O}_{3}$ & 13,55 \\
$\mathrm{P}_{2} \mathrm{O}_{5}$ & $<0,1$ \\
Perda ao Fogo & 19,99 \\
Outros & 0,12 \\
\hline
\end{tabular}

Tabela II - Caracterização química em ppm (análise de traços).

[Table II - Chemical characterization in ppm (small composition).]

\begin{tabular}{cc}
\hline Compostos & Valor (ppm) \\
\hline $\mathrm{Zr}$ & 399 \\
$\mathrm{Mn}$ & 166 \\
$\mathrm{Cr}$ & 177 \\
$\mathrm{Ba}$ & 93 \\
$\mathrm{~Pb}$ & 81 \\
$\mathrm{Sr}$ & 81 \\
$\mathrm{Ga}$ & 65 \\
$\mathrm{Co}$ & 52 \\
$\mathrm{Nb}$ & 64 \\
$\mathrm{Y}$ & 17 \\
$\mathrm{Ni}$ & 12 \\
$\mathrm{Zn}$ & 16 \\
$\mathrm{Cu}$ & $<10$ \\
\hline
\end{tabular}

do rejeito da bauxita, identificando os elementos em maiores e menores concentrações e os elementos traços, respectivamente. Observa-se pela Tabela I que o rejeito ainda apresenta uma quantidade considerável de alumina aproveitável em comparação com a bauxita, pois a bauxita de Paragominas possui teor médio de $50 \%$ de alumina aproveitável [2] e o rejeito contém $21,28 \%$ que é desperdiçada durante o processo de lavagem da bauxita.

A análise de difração de raios X (Fig. 2) identificou as seguintes composições minerais: gibsita, hematita, quartzo e argilominerais como a caulinita. Gibsita, óxidos de ferro e caulinita são os principais coloides responsáveis pelas propriedades de superfície e apresentam uma dupla camada completamente reversível [11].

A Fig. 3 apresenta o resultado da análise granulométrica 


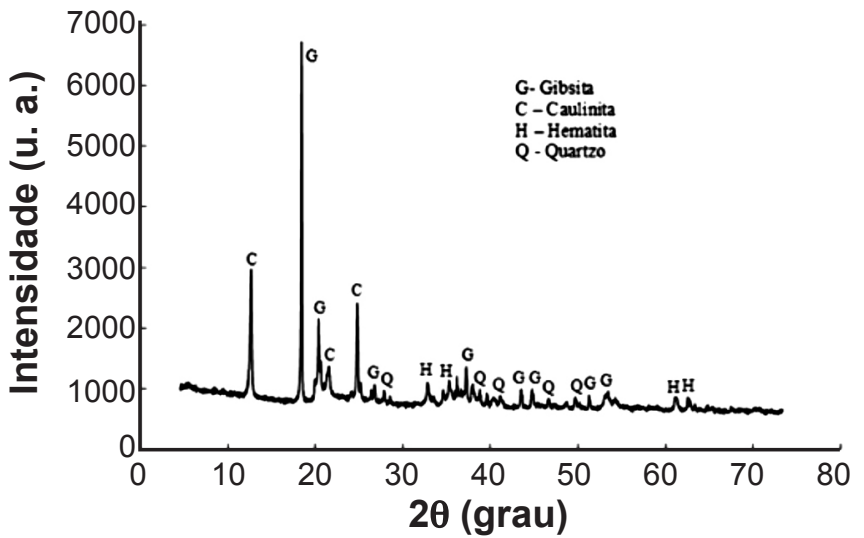

Figura 2: Difratograma de raios X da amostra.

[Figure 2: X-ray diffraction pattern of the sample.]

do rejeito da bauxita. O rejeito pode ser classificado como uma suspensão formada por partículas ultrafinas [12], pois a distribuição granulométrica indicou que cerca de $90 \%$ das partículas possuem tamanhos menores que $0,02 \mathrm{~mm}, 50 \%$ encontram-se abaixo de $0,005 \mathrm{~mm}$ e $22,3 \%$ estão abaixo de $0,002 \mathrm{~mm}$.

A variação de $\mathrm{pH}$ exerce forte influência na viscosidade, pois ela tende a diminuir em $\mathrm{pH}$ ácido e $\mathrm{pH}$ alcalino. A variação de $\mathrm{pH}$ ocasiona o surgimento de cargas elétricas na superfície das partículas. Com isso ocorre repulsão eletrostática como resultado da formação de dupla camada elétrica em torno das partículas [13]. Esse efeito foi observado na Fig. 4, pois o surgimento das cargas elétricas com sua interação repulsiva ocasionou redução da viscosidade em $\mathrm{pH}$ ácidos e alcalinos.

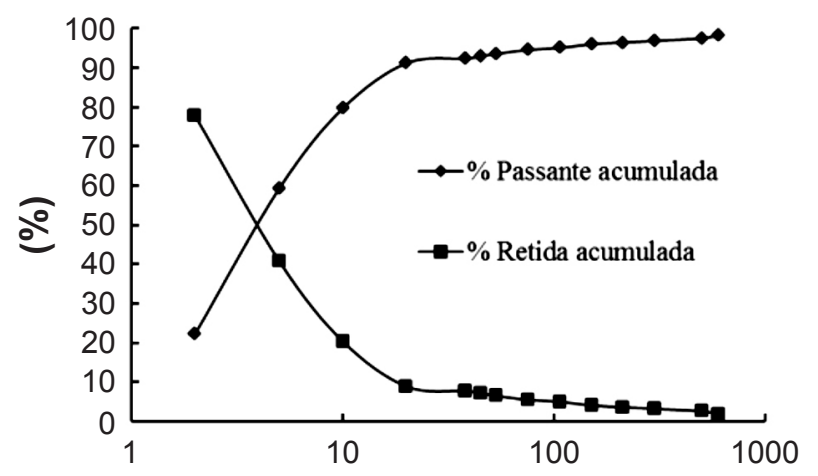

Abertura $(\mu \mathrm{m})$

Figura 3: Curvas de distribuição granulométrica do rejeito da bauxita.

[Figure 3: Particle size distribution curves of tailings of the bauxite.]

A Fig. 5 mostra a defloculação da amostra diante do mecanismo de repulsão eletroestérica, ocasionado pela dupla camada elétrica e pela ação da poliacrilamida $25 \%$ aniônica. As amostras foram analisadas em $\mathrm{pH} 6,5 \mathrm{e} \mathrm{em} \mathrm{pH}$ previamente ajustado para 8,5 . A presença de minerais na amostra que apresentam cargas variáveis dependentes de pH como a caulinita, hematita e gibsita dificulta a avaliação do mecanismo de atuação da poliacrilamida. Esses minerais

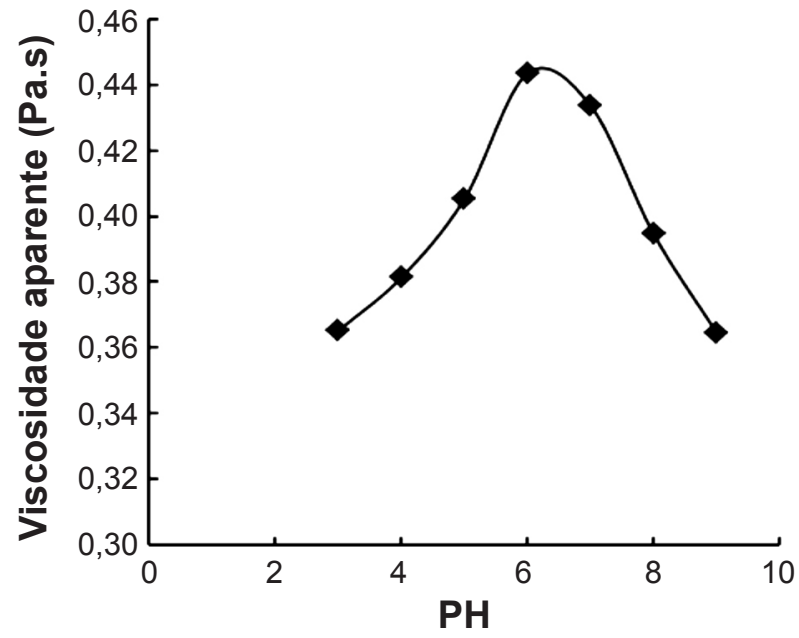

Figura 4: Viscosidade aparente em função do $\mathrm{pH}$. Teor de sólidos $33,5 \%$; taxa - $100 \mathrm{~s}^{-1} /$ tempo - $20 \mathrm{~s}$.

[Figure 4: Apparent viscosity as a function of $\mathrm{pH} 33.5 \%$ of solids content; rate - $100 \mathrm{~s}^{-1} /$ time - $\left.20 \mathrm{~s}.\right]$

apresentam uma dupla camada elétrica completamente reversível e a carga líquida e determinada pelo íon que é adsorvido em excesso [11, 14, 15]. As defloculações (Fig. 5) observadas para os $\mathrm{pHs} 6,5$ e 8,5 podem ter ocorrido devido a uma menor dissociação da poliacrilamida. Os principais fatores a determinar o grau de dissociação de moléculas poliméricas são o pH e a concentração de íons na solução, ou força iônica. Em pHs acima de 8,5 o grau de dissociação dos sítios $\mathrm{RCOOH}$ é alto. À medida que o pH se torna mais ácido o grau de dissociação diminui. Se o grau de dissociação é baixo as cadeias adquirem uma conformação enovelada [7], com isso há a dificuldade de ligação a diferentes partículas e, consequentemente, a solução deflocula. O ponto ótimo de dispersão foi encontrado para a adição de $23,8 \mathrm{~g} /$ ton com redução da viscosidade aparente inicial em 15,61 e 13,70\% para os pHs 6,5 e 8,5 respectivamente.

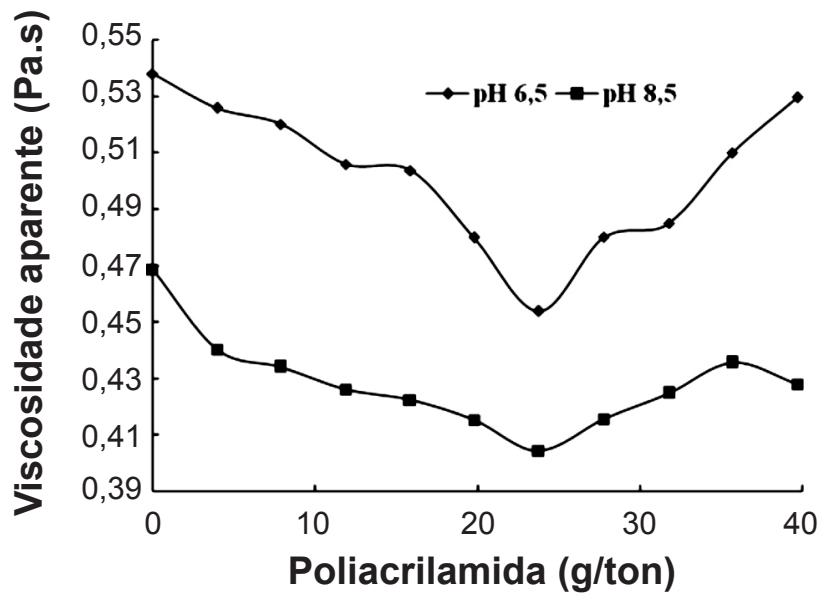

Figura 5: Viscosidade aparente em função da adição de poliacrilamida em pH 6,5 e 8,5 (composição global); teor de sólidos 33,5\%; taxa - $100 \mathrm{~s}^{-1} /$ tempo - $20 \mathrm{~s}$.

[Figure 5: Apparent viscosity as a function of the addition of polyacrylamide in $\mathrm{pH} 6.5$ and 8.5 (global composition); rate $100 \mathrm{~s}^{-1}$ time - $20 \mathrm{~s}$.] 
O mecanismo de repulsão eletroestérica atuou de forma mais eficiente ao ser adicionado no rejeito abaixo da malha 400\# Tyler, com redução em $30,14 \%$ na viscosidade aparente. Observa-se pela Fig. 6 que o ponto ótimo de dispersão encontra-se a 15,9 g/ton, resultado inferior ao obtido na amostra com composição global (Fig. 5). Inicialmente a viscosidade diminui devido à combinação da repulsão eletrostática ocasionado pela dupla camada elétrica originada da maior interação das cargas de superfície das partículas finas e da repulsão estérica causada pela ação do aditivo que interage com as partículas finas que estavam inicialmente emaranhadas, aglomeradas, com orientação ao acaso. Pequenas quantidades de aditivo ao entrar em contato com essas partículas finas, estando sob a ação da taxa de cisalhamento, promove inicialmente a aglomeração de algumas partículas e liberação do líquido que estava retido no interior, com isso a viscosidade diminui. O uso do aditivo em quantidades superiores a 15,9 g/ton liga mais partículas finas em conjunto através de laços e caudas estendidos na solução, aglomerando e floculando a suspensão. Posteriormente, com a adição de excesso de polímero as partículas são restabilizadas por superfície de saturação causando a diminuição da viscosidade.

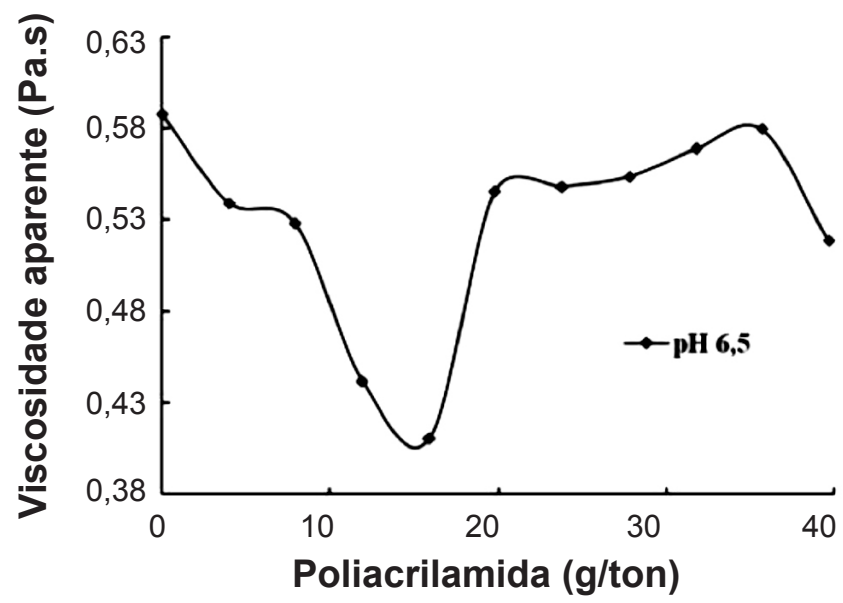

Figura 6: Curva de defloculação com poliacrilamida em $\mathrm{pH}$ 6,5 (passante em 400\# Tyler); teor de sólidos 33,5\%; taxa-100 $\mathrm{s}^{-1} /$ tempo-20 s.

[Figure 6: Defloculation curve with polyacrylamide in $\mathrm{pH} 6.5$ (Passing in 400\# Tyler); rate - $100 \mathrm{~s}^{-1}$ /time - $20 \mathrm{~s}$.]

A adição de diferentes concentrações de hexametafosfato de sódio (Fig. 7) apresentou ponto mínimo com a adição de $101,75 \mathrm{~g} /$ ton, com redução na viscosidade em $8,9 \%$.

Avaliar o comportamento do hexametafosfato de sódio em rejeito de bauxita é difícil devido o mesmo possuir composição química variada. Caulinita, óxidos de ferro e alumínio influenciam nas propriedades de superfície [11] e podem interferir no mecanismo de atuação do hexametafosfato de sódio. Uma das formas de atuação, para o caso da argila, se dá devido os ânions do hexametafosfato de sódio interagir com os átomos expostos de alumínio, surgindo um complexo aniônico [16]. O aumento de carga

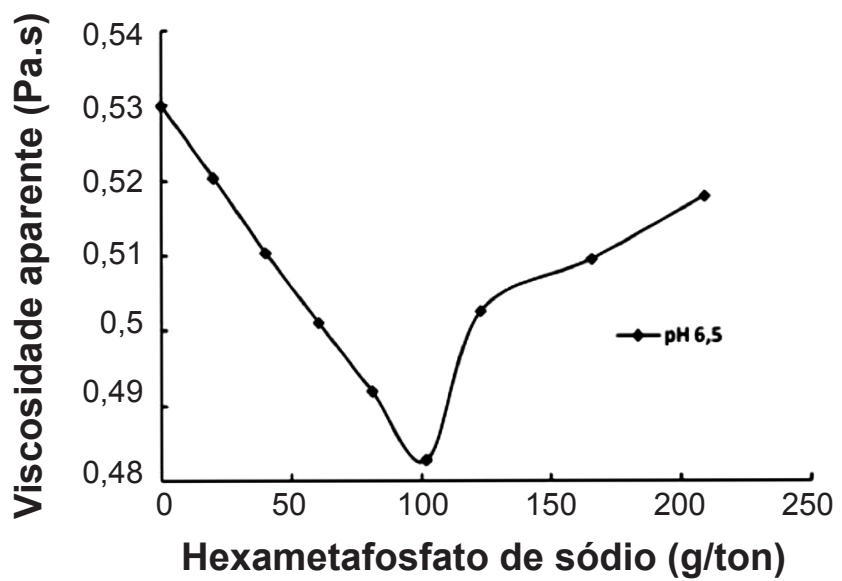

Figura 7: Curva de defloculação com hexametafosfato de sódio em pH 6,5 (composição global); teor de sólidos 33,5\%; taxa-100 s-1/ tempo-20 s.

[Figure 7: Defloculation curve with sodium hexametaphosphate in pH 6.5 (global composition); rate - $100 \mathrm{~s}^{-1} /$ time - $20 \mathrm{~s}$.]

superficial negativa aumenta a força de repulsão entre as partículas. $\mathrm{O}$ outro mecanismo seria a substituição dos cátions floculantes que podem estar dissolvidos por cátions $\mathrm{Na}^{+}$, aumentando assim a espessura da dupla camada elétrica e causando a dispersão [16].

A redução da granulometria (Fig. 8) ao invés de causar consumo mais alto de defloculante devido à tendência natural a aglomeração das partículas coloidais, apresentou efeito contrário, pois reduziu em $13,88 \%$ a viscosidade aparente e o ponto mínimo de defloculação obtido foi para a adição de $60,43 \mathrm{~g} /$ ton, resultado inferior ao encontrado para o rejeito normal (Fig. 7). Isso pode ter ocorrido devido à maior concentração de finos aumentar a área superficial das partículas com consequente aumento nas cargas elétricas, com isso a repulsão eletrostática e a interação partículaaditivo são maiores. Entretanto a menor granulometria ocasionou aumento na viscosidade aparente inicial,

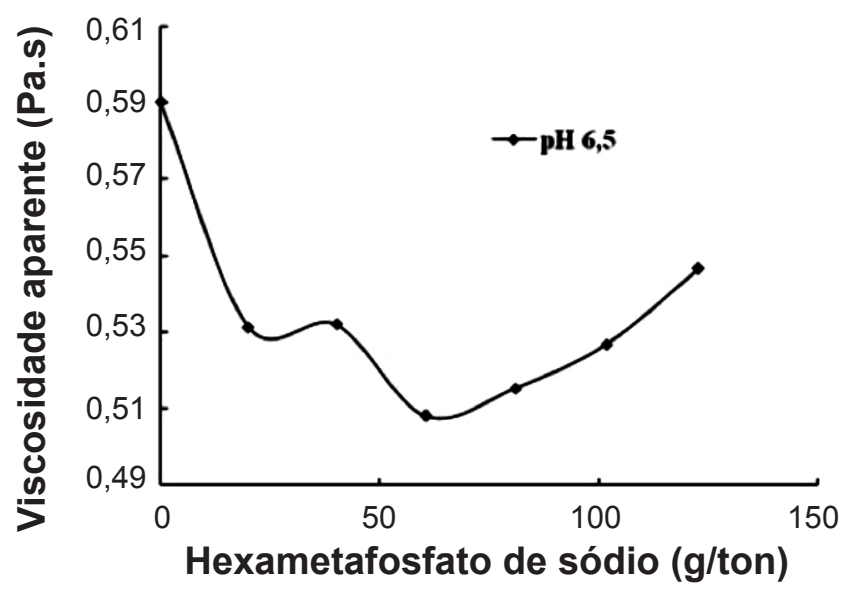

Figure 8: Curva de defloculação com hexametafosfato de sódio em pH 6,5 (passante em 400\# Tyler); teor de sólidos 33,5\%; taxa$100 \mathrm{~s}^{-1} /$ tempo-20 s.

[Figure 8: Defloculation curve with sodium hexametaphosphate in pH 6.5 (passing in 400\# Tyler); rate - $100 \mathrm{~s}^{-1} /$ time - 20 s.] 
consequentemente, o valor obtido no ponto mínimo da amostra passante a 400\# Tyler não foi inferior ao encontrado para a amostra com composição global, tornando o processo desnecessário.

\section{CONCLUSÃO}

Apesar de a poliacrilamida ser indicada para uso como floculante na faixa de pH 6-10 e também, de acordo com a literatura, ser utilizada para esse fim em algumas suspensões minerais, teve comportamento como defloculante na amostra estudada. Observou-se que o comportamento de polímeros aniônicos como floculante ou dispersantes depende da composição química e do controle de $\mathrm{pH}$, pois eles interferem na formação de cargas de superfície, que influenciam o comportamento do aditivo. O melhor resultado de dispersão para a poliacrilamida foi encontrado para $\mathrm{pH} 8,5$, pois o efeito da estabilização eletroestérica foi mais efetivo nesse $\mathrm{pH}$. O hexametafosfato de sódio apresentou comportamento defloculante. Entretanto, o melhor resultado encontrado de dispersão foi para a utilização da poliacrilamida $25 \%$ aniônica. A redução da granulometria potencializou somente a ação da poliacrilamida $25 \%$ aniônica.

\section{AGRADECIMENTOS}

Às empresas Vale e Hydro pelo amostra cedida e ao $\mathrm{CNPq}$ pelo apoio financeiro.

\section{REFERÊNCIAS}

[1] J. J. Varela, Anais Enc. Nac. Tratamento de Minérios e
Metalurgia Extrativa, Porto Alegre, RS 1 (2009) 225.

[2] L. F. Quaresma, Rel. Tec. 22, Ministério de Minas e Energia, Brasil (2009).

[3] A. P. Chaves, Teoria e Prática de Tratamento de Minérios, 2a Ed., Signus Ed., S Paulo, SP (2002) 35.

[4] F. S. Ortega, V. C. Pandolfelli, J. A. Rodrigues, P. A. Sepulveda, Ceram. Ind. 2 (1997) 34.

[5] F. R. C. Chang, G. Sposito, J. Colloid Interface Sci. 178 (1996) 555.

[6] A. Kauppi, K. M. Andersson, L. Bergström, Cem. Concr. Res. 35 (2005) 133.

[7] F. S. Ortega, V. C. Pandolfelli, J. A. Rodrigues, D. P. F. de Souza, Cerâmica 43, 281-282 (1997) 113.

[8] D. E. Abd-El-Khalek, B. A. Abd-El-Nabey, Desalin. 311 (2013) 227.

[9] V. Steven Green, D. E. Stott, Anais $10^{\text {th }}$ Int. Soil Conservation Org. Meet. EUA (1999) 384.

[10] M. J. Hollitt, A. J. Crisp, W. S. Staker, G. M. Roe, D. P. Rodda, US Patent N. 6 (2001) 309.

[11] B. V. Raij, Bragantia, Rev. Cient. Inst. Agronômico Est. S. Paulo 32 (1973) 587.

[12] C. Oliveira, J. Rubio, Série Tecnologia Ambiental 60 (2011) 69p.

[13] J. Cesarano, I. A. Aksay, J. Am. Ceram Soc . 71 (1988) 250.

[14] M. P. F. Fontes, O. A. de Camargo, G. Sposito, Sci. Agricola 58, 3 (2001).

[15] J. Mauri, H. A. Ruiz, R. B. A. Fernandes, J. C. Ker, L. R. M. Rezende, Rev. Bras. Ciência do Solo 35, 4 (2011) 1277.

[16] F. Andreola, E. Castellini, T. Manfredini, M. Romagnoli, J. Eur. Ceram. Soc. 24 (2004) 2113.

(Rec. 10/10/2013, Rev. 02/10/2014, Ac. 31/10/2014) 